Itinéraires Itinéraires

Littérature, textes, cultures

2020-2 | 2020

Discours animal. Langages, interactions, représentations

\title{
Les stéréotypes dans la zoo-anthroponymie française et chinoise
}

Steorotypes in French and Chinese Zooanthroponymy

\section{Lian Chen}

\section{OpenEdition}

\section{Journals}

Édition électronique

URL : https://journals.openedition.org/itineraires/8563

DOI : $10.4000 /$ itineraires.8563

ISSN : 2427-920X

Éditeur

Pléiade

Référence électronique

Lian Chen, "Les stéréotypes dans la zoo-anthroponymie française et chinoise », Itinéraires [En ligne], 2020-2 | 2020, mis en ligne le 23 décembre 2020, consulté le 24 novembre 2021. URL : http:// journals.openedition.org/itineraires/8563 ; DOI : https://doi.org/10.4000/itineraires.8563

Ce document a été généré automatiquement le 24 novembre 2021.

\section{cc) $(9)$}

Itinéraires est mis à disposition selon les termes de la licence Creative Commons Attribution - Pas d'Utilisation Commerciale - Pas de Modification 4.0 International. 


\section{Les stéréotypes dans la zoo- anthroponymie française et chinoise}

Steorotypes in French and Chinese Zooanthroponymy

Lian Chen

\section{Introduction}

1 La phraséologie ${ }^{1}$ est un phénomène linguistique et lexiculturel essentiel, «porteur de l'idiosyncrasie d'une culture, d'une société, d'une façon collective de voir les choses, d'une façon idiomatique de parler» (González-Rey 2002 : 40). Les unités lexicales d'une langue, qui englobent la phraséologie, sont dotées d'une charge culturelle forte. Robert Galisson (1995: 6) définit comme «lexiculture » «la culture mobilisée et actualisée dans et par les mots de tous les discours dont le but n'est pas l'étude de la culture pour elle-même » et souligne les connotations et l'opacité de «mots à charge culturelle partagée (CCP)» $(1987,1989,1991)$. Cette charge culturelle partagée renvoie à des réalités beaucoup plus vastes et complexes que ne le laisserait supposer la définition sémantique des lexies².

La compétence culturelle est constituée d'un ensemble diversifié de représentations partagées, qui sont autant d'images du réel collectif, images le plus souvent réductrices et donc déformantes mais indispensables à la communauté, qui fournissent à ses membres autant (ou presque) de prêt-à-connaître/penser/dire qu'il en est besoin pour le confort (tout relatif bien entendu) de leurs actes de communication. Ces représentations - qui, on le sait, tendent par essence au figement, c'est-à-dire en premier lieu au stéréotypage - participent à des idéologies (de type religieux, économique, politique...) et sont les constituants de base du noyau dur de la compétence de communication. (Boyer 2001 : 334)

2 Élément fondamental de la culture, le langage reflète donc les conditions de vie du peuple et l'histoire de la civilisation dont il émane (Wierzbicka $1988: 2$ ). « Les noms des animaux offrent un terrain d'investigation particulièrement productif car ils sont 
présents dans le [lexique] métaphorique de toutes les langues du monde » (Avenas et Walter 2013). L'humanité préhistorique vivait en étroit contact avec les animaux et «[1]'histoire de l'humanité est d'ailleurs jalonnée de grands bestiaires » (ibid.), comme en témoigne l'art pariétal. Elle a survécu grâce à la chasse puis à l'élevage et à l'agriculture. Certes, l'ère contemporaine s'est éloignée de ce contact avec la nature, mais il n'en reste pas moins que les animaux « habitent » le langage humain, riche en expressions ou idiotismes animaliers.

3 L'imagerie animale est une remarquable source de créativité lexicale, et de nombreuses lexies animalières sont utilisées pour désigner les humains. Catherine Ruchon, Laura Goudet et Marie-Anne Paveau (2018) appellent cet ensemble de dénominations lexicalisées des "zoo-anthroponymes", "définissables comme des zoomorphismes langagiers, en l'occurrence lexico-sémantiques, terme qu'[elles construisent en s'inspirant] de zootoponyme (lieu recevant un nom d'animal), lui-même construit à partir de zoonyme (nom d'animal) » (ibid).

4 L'animal ayant longtemps été considéré comme d'une "essence " inférieure ou fondamentalement différente de celle de l'humain, il n'est pas étonnant que la lexiculture, et en particulier la zooanthroponymie, reflète une sorte de mépris envers celui-ci. L'examen des expressions figées, comparaisons et métaphores, montre que si l'homme reconnaît certaines qualités à l'animal, c'est pour mieux stigmatiser sa stupidité, son ridicule ou ses « turpitudes » présumées.

5 Or, changement important dans les mentalités occidentales après la révolution féministe, depuis les années 1980 une nouvelle attaque est portée à la suprématie de l'humain et à l'anthropocentrisme. L'évolution de la pensée du rapport de l'homme à l'animal, initiée par le philosophe utilitariste australien Peter Singer, associée aux découvertes scientifiques qui se poursuivent sur l'animal, a entraîné la genèse et l'expansion du mouvement antispéciste, qui pose la question de l'animal ou plutôt des animaux en tant que sujets, qui doivent « disposer des mêmes droits que les individus » (Regan et Singer 1976).

6 Mais l'imaginaire collectif s'est construit en même temps que le langage, dans l'ignorance de données scientifiques récentes qui situent dorénavant l'humain et l'animal dans un continuum. Si le langage et le vocabulaire évoluent en même temps que les mentalités, les expressions idiomatiques, si injustifiées puissent-elles paraître de nos jours, restent du fait même de leur figement, à l'écart de ces évolutions.

7 C'est à ces expressions idiomatiques ou «成语chengyu ${ }^{3}$ » en chinois que nous nous intéresserons :

séquences polylexicales [...] qui se caractérisent sémantiquement par leur noncompositionnalité, au moins partielle, qui peut être le résultat d'un procédé tropique (essentiellement la métaphore ou la métonymie). Elles se définissent syntaxiquement par un degré minimal de fixité et lexicalement par une fermeture, au moins partielle, des classes paradigmatiques. (Bolly $2011: 43$ )

Le figement étant un "mécanisme cristallisant l'idiomaticité d'une langue » (Mejri 2008: 245), une telle étude nécessite une double approche, interlinguistique et interculturelle.

8 En écho à notre thèse ${ }^{4}$, nous choisissons ici une approche contrastive entre les lexicultures française et chinoise dans le domaine particulier des expressions idiomatiques mobilisant le champ lexical de l'animal. Il nous semble intéressant, en effet, de comparer les représentations animales dans deux traditions (occidentale/ 
française et orientale/chinoise), et d'analyser comment ces représentations sont influencées par des substrats religieux et philosophiques induisant une conception parfois différente du rapport de l'humain au cosmos en général et à l'animal en particulier.

Notre recherche se fonde ainsi sur deux corpus (français et chinois), construits à partir de dictionnaires monolingues (entre autres: Rey et Chantreau 1989, Lafleur 1991) et bilingues (par ex. Sun 2012) ${ }^{5}$, et analysés selon deux axes (valorisant/dévalorisant), afin de dégager les stéréotypes communs, mais aussi les différences. Parmi les 256 expressions idiomatiques françaises et 530 chengyu relevés ayant pour lexies clés les animaux, nous avons recensé celles qui entrent dans le cadre de la zoo-anthroponymie et caractérisent ou animalisent l'humain : 120 en français, 171 en chinois.

Nous étudierons donc quelles sont les constantes et/ou les différences métaphoriques qui sont associées au registre animalier dans les expressions idiomatiques ${ }^{6}$ des deux langues et cultures. Quel regard sur l'animal est porté en particulier dans la zooanthronymie, ou utilisation du lexique animalier pour désigner l'humain? Le discours humain sur l'animal est-il majoritairement positif, ou stigmatisant et négatif? Se pose d'abord la question de l'arbitraire de ces représentations animales, puis celle du spécisme dans les deux lexicultures, nuancé dans la tradition orientale par l'existence d'animaux valorisés, vénérés et élevés au rang de mythe.

\section{La question de la " motivation »: de l'observation - parfois contestable - à l'arbitraire total}

11 Cette « motivation » concerne l'objectivité et le réalisme des représentations animales.

\subsection{Expressions relativement « motivées » par l'observation}

12 Une petite partie de ces expressions semblent en effet relativement "motivées " par l'observation « réaliste » des animaux sous leur apparence physique, leurs capacités, ou leur comportement. Ainsi, les deux cultures reconnaissent la force du bœuf, animal domestique essentiel à la vie agricole : «être fort comme un bouf » équivaut à 气壮如 牛 (qì zhuàng rú niú, force/puissance+fort+comme+bœuf) ${ }^{7}$ et 高头大马 (gāo tóu dà mă, haut+tête+grand+cheval), désigne une personne de haute taille. De même, la lenteur est associée dans les deux langues à l'escargot : 《avancer comme un escargot », 蜗行牛步 (wō xíng niú bù, escargot+marcher+bœuf+marcher) ${ }^{8}$ : comme rampe l'escargot et marche le vieux bœuf, ni le gastéropode ni le lourd bovin n'incarnant l'aptitude à la vitesse. Autre exemple, en français comme en chinois, une personne qui se contente de répéter ce qu'elle entend, est assimilée à un perroquet : « faire le perroquet », 测鹉学舌 (yīng wǔ xué shé, perroquet ${ }^{9}+$ apprendre+langue). Cette association se justifie par la capacité qu'a cet oiseau de reproduire des sons humains. La fierté et l'orgueil déplacé mêlés à la stupidité sont également incarnés dans les deux langues par le même animal, qui « fait la roue et se pavane »: « être fier comme un paon». En chinois, cette image existe sans toutefois être une expression idiomatique. Enfin, la saleté est associée en français à l'image du porc ou du cochon : "sale comme un cochon ", " manger comme un cochon». Les porcs, par leur appétence à se prélasser dans la boue et leur odeur, 
sont l'objet d'une très mauvaise image dans les deux cultures, même si notre corpus chinois ne comporte pas d'occurrence idiomatique à ce sujet.

L'observation des animaux est populaire et universelle, il est loisible de penser que les mêmes vérités apparaissent sous des formes diverses d'une langue à l'autre. La culture étant la marque de l'humanité, il existe un «fond commun» de représentations animales qui dépasse les différences culturelles et linguistiques et qui s'explique par la similitude des observations réalistes.

14 Autres exemples d'expressions qui semblent relever de l'observation, " une taille de guêpe » valorise en français une taille très fine, par allusion à la morphologie incontestable de cet insecte. À contrario, des jambes trop maigres sont qualifiées de "jambes de coq" en français, les pattes de ce volatile étant en effet dénuées de musculature. En chinois, cette image est portée par deux autres volatiles : 鸠形洁面 (jiū xíng hú miàn, tourterelle+aspect+cygne+visage), qui signifie avoir un visage émacié, et par extension " être maigre comme un clou ", en référence à la finesse de la tête ou du cou de ces oiseaux. De même, l'expression française "sobre comme un chameau " se réfère à la capacité constatée chez cet animal de rester longtemps sans boire.

D'une manière générale, ces expressions qu'on peut qualifier de "motivées" concernent le registre essentiellement physique et comportemental, car plus facilement observable, surtout quand il s'agit d'animaux domestiques vivant au contact des humains. Cependant, la plupart de ces expressions ne présentent pas, en dépit des apparences, de réelle justification scientifique.

\subsection{Expressions apparemment «motivées » mais sans " vérité » scientifique}

16 Ainsi, «avoir un appétit d'oiseau » devrait signifier en réalité que l'on est capable d'avaler quotidiennement jusqu'à deux fois son poids ${ }^{10}$. Biologiquement, cette expression se révèle donc impropre à exprimer la frugalité et constitue une grossière erreur d'origine "anthropomorphique » : c'est par rapport à l'humain que la quantité alimentaire ingérée par un oiseau est infime.

Dans les deux langues, la laideur est fréquemment évoquée par une métaphore animale :

«Laid comme un pou »; « face de rat »

鸡腘龟背 (jī xiōng guī bèi, poulet+poitrine+tortue+dos) et 獐头鼠目 (zhāng tóu shǔ

mù, chevrotain+tête+rat+yeux) désignent aussi une personne laide.

Or, la « charge culturelle partagée » du mot "pou » ( laideur » et "saleté »), pour reprendre l'idée de Robert Galisson, procède d'une analyse évidemment subjective de la réalité, parce que le pou est de taille trop microscopique pour être observé à l'œil nu, donc pour être réputé « laid», et parce qu'il n'est pas interdit de penser que c'est moins le pou qui est «sale » que l'homme dont il se nourrit. Le pou ou le rat ne sont pas plus «laids" que d'autres animaux, ils sont simplement étrangers à la physionomie humaine. Cette qualification est d'ordre esthétique et non scientifique, et ces caractéristiques attribuées aux animaux sont là aussi des clichés anthropomorphiques.

De même, certaines métaphores sont récurrentes pour désigner la férocité ou la cruauté. Elles concernent pour des raisons bien compréhensibles les prédateurs et grands fauves. 
鸱目虎吻 (chī mù hǔ wěn, faucon+yeux+tigre+gueule) évoque une physionomie féroce et cruelle.

鹰膦鹗视 (yīng lín è shì, aigle+fixer+balbuzard+regarder) signifie regarder férocement.

鹰膦虎视 (yīng lín hǔ shì, aigle+fixer+tigre+regarder) désigne un regard féroce et avide.

Concernant ce dernier trait, en français, sans pour autant figurer dans des expressions idiomatiques, la métaphore des «serres» des rapaces ou du «bec d'aigle » pour illustrer la « rapacité » est très prégnante dans les esprits.

Ni le tigre ni l'aigle ne sont « féroces » par nature : ils tuent pour survivre et ne peuvent échapper à leur condition d'animal carnivore qui assure ainsi la survie de son espèce. L'état de nature se situe en effet " par-delà le bien et le mal ». Ces qualifications d'ordre éthique relèvent d'une vision anthropocentrique du monde. La souris déchiquetée par un chat subit probablement des souffrances aussi atroces que l'antilope dévorée vivante par le tigre. En quoi le second est-il plus «cruel» que l'autre? C'est certainement parce qu'ils représentent une menace pour les humains que les grands fauves sont considérés comme féroces. La zoo-anthroponymie est donc le reflet de cette vision partiale et anthropocentrée du monde.

\subsection{L'arbitraire dans les représentations animales}

Le plus souvent, les « qualités » ou « défauts » relèvent de l'imaginaire collectif, de l'a priori, parfois de la littérature. «Faire la mouche du coche » est une allusion littéraire à la fable de La Fontaine Le Coche et la Mouche. Si l'expression désigne une personne qui fait l'indispensable alors qu'elle ne contribue à rien, rien ne permet d'affirmer en pratique que, du point de vue de l'insecte, ce comportement tourbillonnant et bourdonnant, certes agaçant pour l'homme, soit vain. L'être humain peut se révéler « rusé comme un renard». La réputation de cet animal apparaît comme d'origine essentiellement littéraire. Le succès médiéval du Roman de Renart et son machiavélique " goupil », et ultérieurement la fable de Jean de la Fontaine, Le Corbeau et le Renard ont contribué à la généralisation de ce cliché. En chinois, sans être idiomatique, l'expression usuelle 老狐狸 (lăo hú lí, vieux+renard) désigne aussi un homme très rusé, même si la connotation est parfois plus ambiguë, la ruse pouvant se teinter de malhonnêteté ou de perfidie. Dernier exemple, l'ambition attribuée au loup : " être un jeune loup ", " avoir les dents longues ; avoir les dents qui rayent le parquet ", équivalents du chinois : 狼子野心 (láng zǐ yě xīn, louveteau+ambition effrénée).

21 Les expressions mettant en scène des animaux peuvent aussi provenir de légendes antiques, que l'on retrouve ensuite dans plusieurs langues et cultures européennes et moyen-orientales. Il en va ainsi de l'hypocrisie supposée du crocodile («verser des larmes de crocodile »). Le phénomène de mastication activant chez certains sauriens les glandes lacrymales, ceux-ci semblent donc "pleurer » quand ils dévorent une proie. L'expression et la légende proviennent vraisemblablement de cette observation. L'arbitraire est donc ici partiel, comme pour «la mouche du coche »: on ajoute à l'observation d'un comportement physiologique réel une interprétation psychologiquement « anthropomorphique ».

22 Autre exemple d'arbitraire, un même animal peut se retrouver connoté de façon opposée dans les deux langues. Ainsi, l'expression «malin comme un singe » évoque aujourd'hui l'habileté et l'ingéniosité de ce primate, tandis qu'en chinois, 猴头猴脑 
(hóu tóu hóu nǎo, singe+tête+singe+cervelle) fait allusion au comportement irréfléchi et impétueux prêté à l'animal, et devient synonyme de stupidité.

On comprendra facilement pourquoi l'arbitraire de la zoo-anthroponymie concerne plus particulièrement le registre psychologique, intellectuel ou moral. Il s'agit en effet de défauts ou plus rarement de qualités humain'e's attribuée's arbitrairement aux animaux, dans une représentation totalement interprétative de la réalité, ou de l'association d'idées, selon une «logique » peu scientifique et souvent dévalorisante pour l'animal.

\section{La question du spécisme dans les deux lexicultures}

Les individus forment souvent leurs opinions et forgent leurs attitudes selon des représentations. Si ces opinions et attitudes s'écartent d'une base factuelle objective et deviennent des préjugés subjectifs, cette attitude sociale peut aboutir à une forme de discrimination comme le spécisme, "vision du monde postulant une hiérarchie entre les espèces animales et, en particulier, la supériorité de l'être humain sur les $\operatorname{animaux}^{11} »$.

Soulignons d'abord la prévalence dans notre corpus d'expressions dévalorisantes sur l'animal : 79 \% (95 sur 120) en français et quasiment $75 \%$ (128 sur 171) en chinois.

\subsection{Les stéréotypes langagiers : un regard essentiellement négatif sur l'animal}

Dans les deux langues, les références animalières pour désigner l'humain ne sont en général guère flatteuses, ni pour l'un ni pour l'autre. Il nous semble pertinent d'aborder cette analyse selon trois axes : physique et sexuel; cognitif (" l'intelligence» humaine vs la «stupidité » animale); psychologique, comportemental voire «moral». En effet, le physique concerne l'état de "nature » commun à l'animal et à l'humain, tandis que les deux autres relèvent de l'état de "culture", considéré comme prérogative et spécificité humaines, dans une "progression/évolution" où le second est réputé s'éloigner de plus en plus du premier. Les métaphores animales présentes dans les expressions idiomatiques revêtent ainsi souvent une connotation dévalorisante.

\subsubsection{Un physique et une sexualité stigmatisés}

Outre les expressions évoquant la maigreur, la lenteur, la saleté, la laideur déjà citées, de nombreuses autres évoquent négativement l'allure ou le visage. Ainsi, l'éléphant, par sa masse, évoque en français la balourdise et la maladresse : «se comporter comme un éléphant dans un magasin de porcelaine ».

Le profil «aquilin » des rapaces est également connoté négativement dans les deux langues:

鹰嘴鹞目 (ying zuǐ yào mù, aigle+bec+busard+yeux) désigne une personne d'apparence traitre et féroce.

鹰头雀脑 (yīng tóu què năo, aigle+tête+moineau+cervelle) évoque une personne laide et rusée. 
évoquent l'adultère ou la prostitution. Les références aux animaux ne sont ainsi guère valorisantes pour le masculin, dont elles stigmatisent la séduction prétentieuse, la sexualité ou la luxure. Ainsi, le « coq de village/de la paroisse » désigne " un homme qui séduit ou prétend séduire les femmes » (Chollet et Robert $2008: 158$ ). Cette allusion à la basse-cour se retrouve d'ailleurs aussi dans la dénomination très désobligeante de "poule » attribuée aux femmes faciles ou même aux simples compagnes. Un homme volage très porté sur le sexe est qualifié de « chaud lapin » par allusion à la fécondité de cet animal. L'expression usuelle « vieux cochon » est synonyme d'« obsédé » sexuel. En chinois, 狂蜂浪蝶 (kuáng fēng làng dié, folle+guêpe+frivole+papillon) désigne un homme lascif, un coureur de jupons, tout comme en français, un homme qui "papillonne » multiplie les partenaires sexuels.

31 En ce qui concerne les femmes, nous ne nous attarderons pas sur l'image très dévalorisante de la morue ou de la grue (ou prostituée) en français : " être une grue ", "se comporter comme une grue ", ou du poulet en chinois. En effet le sinogramme 鸡 (jī, poulet) est l'homophone de 妓 (jì, prostituée). Notons que les hommes qui se prostituent sont appelés 鸭 (yā, canards), selon une métaphore plus récente.

À contrario, on qualifie d' " oie blanche » une jeune fille candide « qui n'a pas connu le loup» (eu de relations sexuelles). Sans pour autant figurer dans une expression idiomatique, 狼 (láng, loup) évoque la sexualité masculine et 色狼 (sè láng, beauté des femmes+loup) ${ }^{12}$ désigne la salacité ou le satyre. Le chengyu 如狼似虎 (rú láng sì hǔ, comme+loup+comme+tigre) désigne quant à lui, de façon très imagée, le désir dévorant des femmes entre deux âges.

\subsubsection{Un préjugé sur I'« intelligence » humaine vs la « stupidité » animale}

Le lexique animalier stigmatise la faiblesse intellectuelle, la stupidité, la «bêtise ». Les Français moquent ce trait à travers les oiseaux: "avoir une cervelle de moineau ", " une tête de linotte » (être très étourdi), les volatiles (" être une bécasse/dinde ») ou l'âne : « un bonnet d'âne », « un âne bâté ». L'âne et le bœuf en chinois sont également synonymes de stupidité.

34 Les allusions aux animaux évoquent également la naïveté stupide (« une vache à lait », «être le dindon de la farce»), ou encore l'incapacité à apprécier les choses et le manque de discernement: 对牛弹琴 (duì niú tán qín, face+bœuf+jouer+luth), litt. jouer du luth devant un buffle, en français « jeter des perles aux pourceaux » ou « donner de la confiture aux cochons ». 蝉不知雪 (chán bù zhī xuě, cigale+ne...pas+connaître+neige) désigne une personne peu lucide. En effet, la vie éphémère de cet insecte ne lui permet pas de connaître l'ensemble d'une année. Enfin, 鼠目寸光 (shǔ mù cùn guāng, rat+yeux+pouce+lumière) se dit de quelqu'un qui «ne voit pas plus loin que le bout de son nez». 


\subsubsection{Une vision méprisante dans le domaine comportemental et moral}

Dans les deux cultures, les animaux semblent posséder la plupart des défauts et vices humains.

Les expressions idiomatiques françaises condamnent ainsi l'insouciance (« vivre comme l'oiseau sur la branche »), le mutisme (" être muet comme une carpe ») ou au contraire le vain bavardage (" bavard comme une pie »), voire le persiflage (" langue de vipère »). Les reptiles sont associés à la paresse ("paresseux comme une couleuvre»; «faire le lézard »), la mauvaise éducation est considérée comme la spécificité de l'ours (" être un ours mal léché»), tandis que la sévérité ou le manque de loyauté deviennent les attributs de la vache malgré la placidité légendaire de cet animal (« être une peau de vache ", "donner un coup de pied en vache »). Comble de l'ingratitude humaine, le chien (trop) fidèle à l'homme est pour cette même raison méprisé car considéré comme servile ("faire le chien couchant») et acceptant les brimades («traiter quelqu'un comme un chien ») ou " une vie de chien».

En chinois, l'expression non idiomatique associée au chien: 狗腿子 ${ }^{13}$ (gǒu tǔ̌ ž̌, chien+jambe+suffixe marquant un nom) désigne " un laquais, une personne soumise ». Le lexique chinois condamne d'autres traits peu sympathiques dont le chien fait les frais :

人模狗样 (rén mó gǒu yàng, humain+apparence+chien+apparence) se dit d'une personne qui fait semblant d'être sérieuse, mais ne l'est pas.

Un 狼心狗肺 (láng xīn gǒu fèi, loup+cœur+chien+poumon) est un ingrat, un méchant.

Un 狗苟蝇营 (gǒu gǒu yíng yíng, chien+honteux+mouche+chercher) est un individu qui s'est enrichi sans scrupule, tel un chien ou une mouche prêts à tout pour survivre.

Un 狗仗人势 (gǒu zhàng rén shì, chien+s'appuyer sur+humain+puissance) est un faux brave, un imposteur, comme le chien qui mord ou aboie, fort de l'appui de son maître.

L'entêtement («têtu comme une mule ») ou le mauvais caractère ne sont pas oubliés : " un caractère de chien/de cochon », " une tête de cochon ». En chinois l'expression usuelle 猪头 (zhū tóu, litt. « tête de cochon) s'applique à une personne stupide. Dans les deux cas, le constat est néanmoins le même : cette référence à un animal domestique est porteuse d'une connotation péjorative.

L'imagination humaine ne semble pas avoir de limites en ce qui concerne les défauts attribués aux animaux. Dans les deux langues, l'avarice et la mesquinerie sont incarnées par le rat: 《être un rat»; 鼠肚鸡肠 (shǔ dù jī cháng, rat+ventre+poule+intestins) - allusion métaphorique en chinois à "l'étroitesse » de leurs entrailles. La lâcheté est représentée dans cette langue par le même rongeur 抱头 鼠要 (bào tóu shǔ cuàn, se couvrir+tête+rat+s'enfuir) et en français par la «poule mouillée ».

D'autres expressions idiomatiques stigmatisent l'avidité ou la cupidité :

苍蝇见血 (cāng yíng jiàn xuě, mouche+voir+sang) veut dire : avide comme une mouche à la vue du sang.

狼吞虎咽 (láng tūn hǔ yàn, loup+avaler+tigre+avaler) signifie goinfre ou avide.

狼贪鼠窃 (láng tān shǔ qiè, loup+avide+rat+voler) a pour sens : être cupide.

D'une manière plus générale, une meute d'animaux devient synonyme de malfaiteurs :

与狼共舞 (yǔ láng gòng wŭ, avec+loup+ensemble+danser) signifie s'associer à des gens malfaisants. 
狼虫虎豹 (láng chóng hǔ bào, loup+insecte+tigre+léopard) désigne des forbans, des gangsters.

Enfin, 狐群狗党 (hú qún gǒu dăng, renard+groupe+chien+clique) ne saurait évoquer qu'un ramassis de gens sans foi ni loi, des « brebis galeuses ».

Dans un anthropomorphisme langagier, l'être humain attribue aux animaux ses propres défauts et «turpitudes». Notons au passage que si les animaux domestiques font l'objet d'un relatif mépris, les animaux sauvages, eux, sont sources de craintes et sont donc associés à la cruauté et à la férocité, comme souligné en première partie.

\subsection{Quelle explication d'ordre culturel et civilisationnel peut-on apporter à ces représentations animales négatives ?}

L'imaginaire occidental est l'héritage de la philosophie antique et du judéochristianisme. La pensée grecque de Platon et Aristote était une cosmologie considérant l'univers comme "parfait » et "harmonieux", où chaque être vivant avait sa place déterminée. Il s'agissait d'un monde "aristocratique » et hiérarchisé, non seulement entre l'homme et l'animal, mais aussi entre les hommes, même si tous deux étaient pensés comme partie intégrante du cosmos. Selon Hilfiger (2004), les Grecs et leurs disciples les plus modernes jusqu'aux humanismes, ont défini ainsi la spécificité humaine : «l'animal serait un homme insuffisamment logique pour penser le divin et donc être divin à son tour ; l'homme est un animal rationnel ».

Les monothéismes, fondamentalement anthropocentristes, ont introduit une rupture entre hommes et animaux. En faisant de l'homme le sommet de la création, créé à l'image de dieu, ils lui confèrent une nature fondamentalement différente de celle de l'animal, considéré comme inférieur et quantité négligeable. Bien plus, l'animal peut symboliser la faute, le péché (Vincent-Cassy 1984).

Dans sa philosophie mécaniste, Descartes (1596-1650), au XVII siècle, a élaboré contre toute évidence une aberrante théorie de l'animal-machine, incapable d'intelligence, mais aussi de plaisir et de douleur. Il affirme que non seulement « les bêtes ont moins de raison que les hommes, mais qu'elles n'en ont point du tout ${ }^{14} »$. L'être humain, contrairement à l'animal, échappe à son statut de pure machine parce qu'il possède aussi une âme. Cette théorie dualiste de l'âme et du corps a engendré et justifié la conception moderne de l'animal-objet (Chapouthier 2009).

Le spécisme n'est pas totalement absent de la culture chinoise. En effet, bien que celleci soit très influencée par le taoïsme et le bouddhisme, elle hérite aussi de l'influence assez contradictoire du confucianisme. Confucius (551-479 av. J.-C.) prône certes le concept de 仁 rén ou bienveillance envers le vivant, mais il propose parallèlement une vision hiérarchisée de la société humaine et de l'animal.

De ce fait, l'exploitation et la domination des autres espèces animales par l'humain sont justifiables: les animaux ne possèdent ni conscience ni morale, et la plus grande différence entre les humains et les animaux est que les hommes vivent selon les exigences des礼 lǐ (rites ou moralité), qui «sont la plus haute expression de l'ordre hiérarchique » (Watson 1963 : 71) :

Les perroquets peuvent apprendre à parler, mais ce sont des oiseaux après tout ; les singes peuvent apprendre à parler, mais ils appartiennent toujours aux bêtes. Si les hommes ne respectent pas les rites, bien qu'ils sachent parler, leur cœur n'est-il pas celui d'une bête ? [...] Par conséquent, le sage a développé un ensemble de rituels de 
bienséance pour éduquer les hommes, afin qu'ils sachent qu'ils sont différents des

bêtes $^{15}$. (Zhen et Kong [1222-1696] 2004 : volume 曲礼 Qū lì)

Parmi ces rituels figurent les sacrifices d'animaux. Dans la philosophie confucéenne, la vie animale importe moins que le maintien des règles et des traditions féodales, l'intérêt social primant sur tout autre considération.

\section{Quelques images valorisantes malgré tout en zoo- anthroponymie}

Les expressions animalières valorisantes sont minoritaires dans notre corpus, mais pas absentes : $21 \%$ (25 sur 120 ) en français, $25 \%$ (43 sur 171$)$ en chinois.

\subsection{Représentations positives dans les deux cultures}

\subsubsection{Clichés physiques}

Est ainsi valorisée la force masculine : «fort comme un taureau »; 气壮如牛 (qì zhuàng rú niú, puissance+fort+comme+bœuf); 虎背熊腰 (hǔ bèi xióng yāo, tigre+dos+ours+taille). Mais l'évocation animale ne saurait se révéler totalement laudative. Cette force a en effet pour revers une faiblesse intellectuelle : « un bœuf » est aussi un individu obtus ou d'allure grossière.

Une silhouette fine ou la grâce sont des critères positifs pour le physique féminin :

«taille de guêpe »

惊鸿游龙 (jīng hóng you lóng, formidable+oie sauvage+nager+dragon) associe la grâce à « une oie sauvage qui vole ou un dragon qui nage ».

网若惊鸿 (piān ruò jīng hóng, s'envoler+comme+formidable+oie sauvage) désigne aussi la grâce d'une danseuse.

\subsubsection{Quelques qualités d'intelligence ou de comportement sont aussi « reconnues » dans les représentations animales}

Ainsi, « une fine mouche » ou " pas folle la guêpe » s'appliquent aux personnes douées d'une intelligence subtile. En français, «être une vraie fourmi » est plutôt positif et souligne le sens de l'économie. Dans les deux langues existent des associations proches entre animaux et certaines qualités.

Le courage est symbolisé dans la lexiculture française par le lion, considéré comme le roi des animaux (« avoir un cœur de lion »). En chinois, les références au lion sont plus rares, cet animal ayant été introduit tardivement sur le territoire. C'est l'expansion du bouddhisme à partir de l'Inde, qui a introduit en Chine l'image du lion, incarnation du courage et de la justice. En chinois d'autres animaux évoquent le courage : 熊心豹胆 (xióng xīn bào dăn, ours+cœur+léopard+vésicule biliaire). La fidélité, quant à elle, est incarnée de façon tout à fait compréhensible par le chien, dont la connotation est donc parfois positive: 犬马之心 (quǎn mǎ zhī xīn, chien+cheval+de+cœur), fidélité totale. Enfin, les ovins sont associés à l'idée de douceur. Cette comparaison est commune aux deux cultures («doux comme un agneau»), avec une connotation positive peut-être plus accentuée en Chine, le français y associant une idée de passivité et de soumission (" être un mouton»). Cependant, la langue chinoise ne l'exprime pas sous forme idiomatique. 
53 La vision de l'animal témoigne cependant de différences significatives entre les lexicultures française et chinoise, dans laquelle on constate un symbolisme animalier bien plus valorisant qu'en français. En témoigne l'existence d'animaux vénérés et élevés au rang de mythe.

\subsection{Une spécificité lexiculturelle : les animaux mythifiés} l'image est très positive en chinois, contrairement à « un usage métaphorique du nom de la grue qui semble être propre au français » (Avenas et Walter 2013). Cet animal présente des occurrences fréquentes dans la lexiculture et l'iconographie chinoises. Tout d'abord, la blancheur de son plumage symbolise la pureté, le raffinement, si bien que cet animal incarne les gens de qualité, l'élite :

Une 鹤立鸡群 (hè lì jī qún, grue+être debout+poules+groupe), une grue au milieu des poules, est une personne d'une remarquable supériorité.

鹤鸣九悬 (hè míng jiǔ gāo, grue+crier+neuf+hauteur) : quand la grue crie au milieu des marais, sa voix s'entend jusqu'au neuvième ciel ${ }^{18}$, signifie qu'un homme distingué aura beau se cacher, il sera tout de même reconnu.

En outre, cet oiseau est réputé de grande longévité. 朱颜鹤发 (zhū yán hè fà, vermillon+visage+grue+cheveux) se dit d'une personne âgée en forme et de bonne allure. Cette image est très valorisante, l'âge étant synonyme d'expérience et de sagesse. 

Qu'il s'agisse du statut de l'homme, de la femme et de l'animal, le lexique - qui « est par 
définition, à l'image du monde qu'il informe et qu'il désigne » (Galisson $1991: 157)$ - se constitue en dépositaire de contenus culturels (Galisson 1999). Comme le souligne la linguiste Anna Wierzbicka, «le langage ne reflète pas directement le monde : il reflète la conceptualisation humaine, l'interprétation humaine du monde ${ }^{21}$ ", ce dont témoigne la zoo-anthroponymie, ou animalisation lexicale de l'humain.

D'une part, celle-ci relève d'un certain arbitraire anthropomorphique, selon une « logique » peu scientifique. D'autre part, elle se révèle majoritairement stigmatisante. La comparaison de deux corpus d'expressions idiomatiques chinoises et françaises montre en effet un certain consensus négatif sur l'animal, reflet d'une vision spéciste et hiérarchisée du vivant.

L'humain, dans son évolution, se distingue de l'animal par son éloignement de l'état de nature vers un état de culture, qui suppose la domination, voire la négation, de sa composante animale. La lexiculture animalière stigmatise donc essentiellement la proximité avec l'état de nature (la force physique brute, la saleté, la « bestialité » ou la "bêtise ») pour valoriser en contrepoint l'état de culture de l'être humain, "animal pensant ", être "intelligent » au sens étymologique, capable de penser le monde, de se poser en sujet et en objet, et doté de conscience et de morale.

On constate cependant dans la lexiculture chinoise un symbolisme animalier plus valorisant qu'en français, lié à l'influence du bouddhisme et du taoïsme. Certains animaux font même l'objet d'un culte, dans une représentation parfois totalement opposée à celle de la culture française.

Héritière de la philosophie gréco-romaine et du judéo-christianisme, la pensée occidentale dissocie l'homme de l'animal et de la nature, alors que pour l'essentiel la pensée orientale les solidarise dans un même univers. Mais aujourd'hui, cette pensée occidentale évolue vers une vision plus harmonieuse et intégrative de l'humain au reste du vivant, intégrés dans un tout ou dans un continuum.

71 Cependant, les représentations animales dans les expressions idiomatiques, du fait même de leur figement, sont le reflet d'une lexiculture issue du passé, et de mentalités qui évoluent moins vite que les découvertes scientifiques et les remises en cause philosophiques récentes.

72 "C'est en tant que pratique sociale et produit socio-historique que la langue est toute pénétrée de culture. Le jeu de symbiose dans lequel fonctionnent langue et culture fait qu'elles sont le reflet réciproque et obligé l'une de l'autre» (Galisson 1988: 330). L'étude de la lexiculture et des expressions idiomatiques animalières en est une illustration et la confirmation.

\section{BIBLIOGRAPHIE}

Association bouddhiste électronique chinoise, 2002, « Das Nirvana-Sutra 大般涅槃紃 Dà bān niè pán jīng », volume VII. Chine, Association bouddhiste électronique CBETA, [En ligne], http:// buddhism.lib.ntu.edu.tw/BDLM/sutra/chi_pdf/sutra7/T12n0375.pdf, consulté le 15 juin 2020. 
Avenas, Pierre et Walter, Henriette, 2013, « Des noms d'animaux dans le vocabulaire des métiers... et d'un nouveau type de "dictionnaire" ", Éla. Études de linguistique appliquée , n 171, p. 287-296, [En ligne], https://doi.org/10.3917/ela.171.0287.

Boyer, Henri, 2001, «L'incontournable paradigme des représentations partagées dans le traitement de la compétence culturelle en français langue étrangère ", Éla. Études de linguistique appliquée , nº 123-124, p. 333-340, [En ligne], https://doi.org/10.3917/ela.123.0333.

Chapouthier, Georges, 2009, « Le statut philosophique de l'animal : ni homme, ni objet », Le Carnet Psy, no 139 , p. 23-25.

Cheng, Anne, [1997] 2005, Histoire de la pensée chinoise, Paris, Seuil.

Collectif, [2005], Dictionnaire du lexique chinois moderne (cinquième édition) 现代汉语词典第五版 Xiàn dài hàn yǔ cí diǎn, Institut de linguistique et académie chinoise des sciences sociales

Bolly, Catherine, 2011, Phraséologie et collocations. Approche sur corpus en français L1 et L2, Bruxelles, New York, Peter Lang.

Confucius, [551-479 av. J.-C.], « Les Entretiens », Chapitre XVII, [En ligne], http:// www.readers365.com/lunyu/index.htm, consulté le 15 juin 2020.

Descartes, René, [1637] 2011, Discours de la Méthode, Édition électronique (ePub) vol. 1,0 : Les Échos du Maquis, p. 35, https://philosophie.cegeptr.qc.ca/wp-content/documents/Discours-de-lam \%C3 \%A9thode.pdf, consulté le 15 juin 2020.

Galisson, Robert, 1987, « Accéder à la culture partagée par l'entremise des mots à C.C.P. », Éla. Études de linguistique appliquée, $\mathrm{n}^{\circ}$ 67, p. 119-140.

Galisson, Robert, 1988, «Cultures et lexicultures. Pour une approche dictionnairique de la culture partagée », dans Cahiers d'études hispaniques médiévales, vol. 7, p. 325-341.

Galisson, Robert, 1989, « Enseignement et apprentissage des langues-cultures - “évolution” ou "révolution" pour demain?", Éla. Études de linguistique appliquée, n 79, p. 35-52.

Galisson, Robert, 1991, De la langue à la culture par les mots, Paris, CLE International.

Galisson, Robert, 1995, « Où il est question de lexiculture, de cheval de Troie, et d'impressionnisme... ", Éla. Études de linguistique appliquée, nº 97, p. 5-14.

Galisson, Robert, 1998, Éla. Études de linguistique appliquée, nº 109, « De l'éthique en didactique des langues étrangères » (coordonné avec Christian Puren).

Galisson, Robert, 1999a, «La pragmatique lexiculturelle pour accéder autrement à une autre culture, par un autre lexique ", Éla. Études de linguistique appliquée, nº 116, p. 477-496.

Ge, Hong, 283-343, « Dui su », dans Baopuzi, Chinese Text Project, 2006, [En ligne], https:// ctext.org/baopuzi/dui-su/ens, consulté le 15 juin 2020.

Goudet, Laura, Paveau, Marie-Anne et Ruchon, Catherine, 2018, « Zoo-anthroponymes. Quand l'animal est le nom de l'humain », Realista, 18 février 2018, [En ligne], https:// realista.hypotheses.org/1581, consulté le 15 juin 2020.

González-Rey, Isabel, 2002, La phraséologie du français, Toulouse, Presses Universitaires du Midi. Hilfiger, Mathieu, 2004, « "L'humanité" chez Platon », dans Le Philosophoire, $\mathrm{n}^{\circ}$ 23, p. 166-194, [En ligne], https://doi.org/10.3917/phoir.023.0166.

Larousse en ligne, « spécisme », https://www.larousse.fr/dictionnaires/francais/ sp\%C3\%A9cisme/188309, consulté le 15 juin 2020. 
Les cent quatre-vingts commandements stipulés par Sieur Lao (老君說一百八十戒 Laojun shuo yibaibashi jie), 2006, [En ligne], https://ctext.org/wiki.pl?if=gb\&chapter=405117\&remap=gb, consulté en juin 2020.

Liu, Jiexiu, 1985, Le chengyu [成语], Pékin, Shang wu yin shu guan.

Ma, Guofan, 1978, Les chengyu 成语, Hohhot, Nei Menggu renmin chubanshe.

Mejri, Salah, 2008, « Figement et traduction : problématique générale », Meta :journal des traducteurs, vol. 53, $\mathrm{n}^{\circ}$ 2, p. 244-252, [En ligne], https://doi.org/10.7202/018517ar.

Pruvost, Jean, 1999, « Lexique et vocabulaire : une dynamique d'apprentissage », Éla. Études de linguistique appliquée, $\mathrm{n}^{\circ} 116$, p. 395-419.

Pruvost, Jean, Lino, Maria-Teresa et Bonicel Lætitia, 2003, Mots et lexiculture : hommage à Robert Galisson, Paris, Champion.

Regan, Tom et Singer, Peter, 1976, Animal Rights and Human Obligations, Englewood Cliffs, NJ, Prentice-Hall.

Sun, Weizhang, 1989, La phraséologie du chinois [汉语熟语学hànyǔ shúyǔ xué], Changchun, Jilin éducation.

Sabban, Françoise, 1979, « La fonction crée-t-elle le proverbe ? Quelques remarques sur les idiomes du chinois moderne ", Cahiers de linguistique - Asie orientale, $\mathrm{n}^{\circ} 6,29-47$.

Sabban, Françoise, [1980] 1999, Idiotismes quadrisyllabiques en chinois moderne, Paris-Hong Kong, Langages Croisés.

Shi, Shi, 1979, Étude du chengyu [汉语成语研究 Hàn yǔ chéng yǔ yán jiū], Chengdu, Si chuan ren min chu ban she.

Tao, Yuanming (auteur original), [365-427] 2012, À la recherche des dieux (Sou shen hou ji), trad. Bao Gan, Shanghai, volume I, site Poésie ancienne, [En ligne],http://www.gushicimingju.com/ dianji/soushenhouji/5433.html, consulté le 15 juin 2020.

Trésor bouddhiste, Vajrayāna, beita, 2008, [En ligne], http://www.beita.org/html/zhengfacang/ D2shenminghaihui/200811/19-1259.html, consulté le 15 juin 2020.

Vincent-Cassy, Mireille, 1984, « Les animaux et les péchés capitaux : de la symbolique à l'emblématique », dans Actes des congrès de la Société des historiens médiévistes de l'enseignement supérieur public, $\mathrm{n}^{\circ} 15, \mathrm{p} .121-132$.

Watson, Burton, 1963, Hsun Tzun Basic Writing, New York, Columbia University Press.

Wierzbicka, Anna, 1988, The Semantics of Grammar, Amsterdam, Philadelphie, John Benjamins Publishing Company.

Wen, Duanzheng, 2006, Cours de lexique du chinois [汉语词汇学教程 Hàn yǔ cí huì xué jiào chéng], Commercial Press.

Zhen, Xuan et Kong, Yinda (auteurs originaux), [1222-1696] 2004, 礼记 Liji (Mémoires sur les rites), mis en forme par Lu, Youren, volume 曲礼Qū lǐ, Shanghai, [En ligne], http://www.zggdwx.com/ lijizhengyi/1.html, consulté le 15 juin 2020.

\section{Corpus}

Ashraf, Mahtab et Miannay Denis, 1995, Dictionnaire des expressions idiomatiques, Librairie Générale Française. 
Centre de recherche du dictionnaire shuo ci jie zi, 2013, Dictionnaire d'expressions idiomatiques chinoises [中华成语大词典 zhōng huá chéng yǔ dà cí diăn], Pékin, Shang wu yin shu guan.

Cai, Hongbin, 2014, Dictionnaire explicatif des expressions et locutions françaises [法语成语解析词典 fãyǔ chéngyǔ jiěxī cídiǎn] Pékin, Shang wu yin shu guan.

Chollet, Isabelle et Robert, Jean-Michel, 2008, Les expressions idiomatiques, Paris, CLE international.

Doan, Patrick et Weng, Zhongfu, 1999, Dictionnaire de chéngyǔ : idiotismes quadrisyllabiques de la langue chinoise [汉语成语词典 hàn yǔ chéng yǔ cí diǎn], Paris, éditions Librairie You-Feng.

Lafleur, Bruno, 1991, Dictionnaire des locutions idiomatiques françaises, Paris, Duculot.

Rat, Maurice, [1957] 2007, Dictionnaire des Expressions et locutions traditionnelles, Paris, Larousse.

Rey, Alain et Chantreau, Sophie, [1989] 2003, Dictionnaire des expressions et locutions, Paris, Le Robert.

Sun, Qian, [1999] 2012, Nouveau dictionnaire chinois-français des locutions et proverbes [新编汉法成语 词典 Xīn biān hàn fã chéng yǔ cí diǎn], Xiamen, Xiamen da xue chu ban she.

Université de Pékin, [1979] 1980, Dictionnaire chinois-français des locutions et proverbes [汉法成语手 册 Hàn fă chéng yǔ shǒu cè], Maison d'Édition de Beijing, Pékin (1979), Hong Kong (1980).

Expressio.fr, https://www.expressio.fr, consulté le 15 juin 2020.

\section{NOTES}

1. En chinois, le terme le plus répandu pour désigner ces figements est «熟 语 shú yǔ : unité phraséologique » (Sun $1989: 1$ ).

2. Nous préférons le terme " lexie » à celui de « mot », terme ambigu en linguistique.

3. Les « sinismes » (Doan et Weng 1982), ou «idiotismes quadrisyllabiques » (Sabban 1980), ont pour forme basique un rythme quaternaire (quadrisyllabique), fixe, divisé phonétiquement et/ou syntaxiquement en deux hémistiches. Cette forme conventionnelle et inchangée depuis des générations s'appelle chengyu, "expression toute faite ». Un chéngyŭ est souvent une expression issue de la littérature classique, utilisée en chinois moderne comme une lexie composée. Il appartient au style élégant et concis, et contient fréquemment un fort contenu allusif (Ma 1978, Shi 1979, Liu 1985, Wen 2006).

4. Sujet de thèse : "Analyse comparative des expressions idiomatiques en chinois et en français relatives au corps humain et aux animaux ».

5. Voir la section corpus en bibliographie.

6. Le cas échéant, nous enrichirons nos exemples de quelques autres stéréotypes langagiers non idiomatiques.

7. Nous adoptons le format suivant : quadrisyllabe, pinyin (prononciation romanisée), sens de chaque sinogramme, sens du chengyu ou équivalent idiomatique français.

8. Les chengyu possèdent de très fortes contraintes métriques et syntaxiques, liées à une tradition rhétorique remontant aux premiers écrits poétiques. On rencontre ainsi dans un grand nombre d'entre eux une structure quadrisyllabique et un "parallélisme » ou "symétrie », lorsque les deux premiers sinogrammes expriment un sens parallèle, sinon similaire, à celui que dénotent les deux derniers (Sabban 1979). Les deux 
dissyllabes présentent une structure syntaxique interne identique, entretenant entre eux un lien de synonymie ou d'antonymie, et/ou les caractères peuvent être mis en correspondance respectivement. Cette structure récurrente des chengyu permet d'en renforcer le sens, et d'assurer un rythme harmonieux. La référence fréquente à deux animaux dans la même expression participe de cet effet stylistique et de ce renforcement sémantique.

9. Ici, les deux premiers sinogrammes forment une seule lexie : 鹦鹉 yīng wǔ. Il en sera de même dans d'autres exemples, ce qui explique la traduction en trois lexies et non quatre en français.

10. Cf. https://www.expressio.fr/expressions/avoir-un-appetit-d-oiseau-mangercomme-un-moineau.

11. Larousse en ligne.

12. Dictionnaire du lexique chinois moderne (cinquième édition), p. 1178.

13. Dictionnaire du lexique chinois moderne (cinquième édition), p. 482.

14. Descartes, Discours de la Méthode.

15. Texte original : 鹦州能言, 不离飞鸟; 猩猩能言, 不离离兽。今人而无礼, 虽能 言，不亦兽兽之心乎？[...] 使人以有礼，知自别于恖兽。

16. Cependant, Nuwa (女娲 nŭ wā), déesse créatrice au corps de serpent et symbole de fertilité, est une figure positive. Il en est de même du signe zodiacal du serpent, tous les signes possédant une valeur associée. Le serpent dans le zodiaque symbolise la beauté, la grâce et la souplesse, etc.

17. Trésor bouddhiste, Vajrayāna.

18. Dans les croyances chinoises, le ciel est constitué de neuf couches, la neuvième étant évidemment la plus haute.

19. Tao Yuanming [365-427] 2012 : volume I.

20. 一切众生皆有佛性yīqiè zhòngshēng jiē yǒu fú xìng.

21. Cité par Pruvost, Lino et Bonicel (2003: 11).

\section{RÉSUMÉS}

Dans toute langue, le lexique est le reflet d'une société et d'une culture qui donnent un statut spécifique à l'homme, à la femme et à l'animal. L'étude des stéréotypes lexicaux « animalisant » l'humain est révélatrice d'un certain anthropocentrisme, dans les zoo-anthroponymies chinoise et française, malgré quelques différences, liées au substrat philosophique et religieux. C'est ainsi qu'un certain spécisme se manifeste dans les expressions idiomatiques des deux langues. Notre étude se base sur un corpus d'expressions idiomatiques françaises et chinoises relevant de la zooanthroponymie, établi à partir de dictionnaires spécialisés en chinois et français.

In any language, the lexicon is the reflection of a society and a culture that gives a specific status to men, women and animals. The study of lexical stereotypes "animalising" humans, reveals a kind of anthropocentrism in Chinese and French zoo-anthroponymies, in despite of some 
differences linked to the philosophical and religious substratum. Thus and so some speciesism is manifested in the idiomatic expressions of the two languages. Our study is based on a corpus of French and Chinese idiomatic expressions relating to zoo-anthroponymies, established from specialized Chinese and French dictionaries.

INDEX

Mots-clés : phraséologie contrastive, lexiculture, expressions idiomatiques, zoo-anthroponymie, chinois, français, spécisme

Keywords : contrastive phraseology, lexiculture, idiomatic expressions, zoo-anthroponymy, Chinese, French, speciesism

\section{AUTEUR}

\section{LIAN CHEN}

Université de Cergy-Pontoise, Laboratoire Langues, Textes, Discours, Dictionnaires (EA 7518 LT2D), associée au laboratoire Dynamiques, Interactions, Interculturalités Asiatiques (D2IA), Université de La Rochelle 